\title{
BALANÇO HÍDRICO NO POTENCIAL DOADOR DE MÚLTIPLOS ÓRGÃOS EM CENTRO DE REFERÊNCIA DE TRAUMA
}

\author{
Balance of fluids in potential multiple organ donors at a trauma referral center \\ Maria Fernanda Spinelli Moura, Elizandra Cássia da Silva, Luiz Eduardo Rafael Moutinho, \\ Olival Cirilo Lucena da Fonseca Neto
}

\section{RESUMO}

Introdução: A abordagem agressiva e efetiva ao potencial doador beneficia quantitativamente e qualitativamente o transplante de múltiplos órgãos. Diante das alterações fisiológicas da morte encefálica, o equilíbrio hemodinâmico protagoniza na otimização desse grupo. Objetivo: Avaliar o balanço hídrico e outros parâmetros do perfil hemodinâmico de pacientes diagnosticados com morte encefálica na UTI com relação ao contexto clínico do transplante de múltiplos órgãos. Métodos: Estudo descritivo, retrospectivo e transversal dos pacientes diagnosticados com morte encefálica, durante 2013 a 2014 , na UTI do centro de referência em trauma do estado de Pernambuco. Os dados foram extraídos de modo sistematizado dos prontuários dos pacientes e as variáveis do perfil geral, clínico e hídrico sintetizadas através da frequência absoluta e relativa. Resultados: De 41 diagnósticos de morte encefálica, 34 potenciais doadores foram analisados, dos quais 15 (44\%) efetivaram a doação de órgãos. O traumatismo cranioencefálico, o sexo masculino e a faixa etária de 41 à 50 anos foi predominante no grupo. Os órgãos mais captados para doação foram rins e fígado, e não houve transplante pulmonar. Todos estavam em uso de drogas vasoativas e o balanço hídrico foi altamente positivo, tanto no primeiro quanto no segundo dia de avaliação do potencial doador falecido. Conclusão: Apesar da hiper-hidratação, a efetivação dos transplantes tem resultado acima da média neste centro. A permissividade do balanço hídrico fortemente positivo é justificado pela ausência de centro especializado no transplante pulmonar na região. O controle rigoroso do perfil hemodinâmico do potencial doador de órgãos aumenta a viabilidade do transplante e deve ser adaptado e sistematizado à realidade de cada localidade.

Descritores: Obtenção de Tecidos e Órgãos; Transplante; Morte Encefálica.

Instituição:

Hospital da Restauração Governador Paulo Guerra, Recife/PE Brasil

\section{Correspondência:}

Olival Cirilo Lucena Fonseca Neto.

R. Jacobina, $n^{\circ} 45-$ Graças - Recife/PE

Tel. (81) 99989-0208

E-mail: olivalneto@globo.com

\section{INTRODUÇÃO}

O transplante de órgãos é uma terapia determinante para o paciente portador de disfunção orgânica terminal. Diante do cenário atual da necessidade de transplantes, - mais de 30 mil pacientes ativos na lista de espera - a otimização do potencial doador falecido é essencial. ${ }^{1}$

A morte encefálica resulta em alterações fisiológicas que conduzem à falência múltipla de órgãos, fenômeno potencialmente grave ao transplante. ${ }^{2}$ Esse contexto demanda uma assistência clínica especializada e multiprofissional, reservada à Unidade de Terapia Intensiva (UTI). ${ }^{3,4}$ A abordagem agressiva e efetiva ao potencial doador beneficia quantitativamente $e$ qualitativamente os órgãos disponíveis para doação. ${ }^{5,6}$

Vários mecanismos são responsáveis pela lesão orgânica sistêmica na morte encefálica, entretanto o equilíbrio hemodinâmico protagoniza nesse cenário. ${ }^{6,7}$ 
A má perfusão e inadequada oxigenação periférica correspondem à série final de uma sequência complexa de fenômenos autônomos e inflamatórios que, inicialmente, manifestam hipertensão arterial transitória, seguida por um estado persistente de choque circulatório. ${ }^{2,8}$ Os distúrbios endócrinometabólicos podem contribuir para depleção do volume circulante efetivo (a diabetes insipidus central procede em $46-86 \%$ dos casos) acentuando a hipovolemia e a hipernatremia. ${ }^{7}$ Nesse contexto, diferentes estratégias vêm sendo estudadas para adequar o balanço hídrico na preservação de múltiplos órgãos. ${ }^{9-11}$

O presente estudo objetiva avaliar o balanço hídrico e outros parâmetros do perfil hemodinâmico de pacientes diagnosticados com morte encefálica na UTI em relação ao contexto clínico do transplante de múltiplos órgãos.

\section{MÉTODOS}

Este estudo realizou uma análise descritiva, retrospectiva e transversal dos potenciais doadores de órgãos diagnosticados com morte encefálica, durante o período de janeiro de 2013 a 31 de dezembro de 2014, na UTI do centro de referência em traumatologia e neurologia/ neurocirurgia - Hospital da Restauração Governador Paulo Guerra - do estado de Pernambuco, Brasil.

Os dados referentes ao sexo, idade, motivo da internação, resultado da doação, órgãos doados, perfil hemodinâmico (uso de drogas vasoativas), perfil renal (creatinina e ureia), perfil hepático [transaminase glutâmica oxalacética (TGO), transaminase glutâmica pirúvica (TGP), fosfatase alcalina (FA), gama glutamil transferase (GGT), razão normalizada internacional (INR), perfil eletrolítico (sódio sérico e potássio) e do balanço hídrico (corrigido pelo peso) foram extraídos a partir do dia de abertura do protocolo de morte encefálica e estruturados em um questionário desenvolvido para esta pesquisa. Pacientes com sorologia reagente para doenças infectocontagiosas e diagnosticados com neoplasia e sepse durante o período proposto foram excluídos.

Os perfis geral, clínico e hídrico foram sumarizados, respectivamente, nas Tabelas 1, 2 e 3 . A frequência relativa dos órgãos doados foi comparada na Figura 1.

O estudo considerou as referências, de acordo com recomendação do laboratório local, para avaliar o desfecho do grupo estudado.

Para a análise dos dados, foi utilizada a distribuição de frequências das variáveis pesquisadas, através do IBM Statistical Package for Social Sciences (SPSS) versão 22.0, que viabilizou o cálculo das medidas descritivas de frequência absoluta e frequência relativa (\%).
A pesquisa cumpriu com os princípios enunciados na resolução 466/12 do Conselho Nacional de Saúde, com registro de apreciação e aprovação (CAAE: 47866015.0.0000.5198) pelo Comitê de Ética, em Pesquisa do hospital em questão.

Figura 1: Distribuição comparativa dos órgãos doados

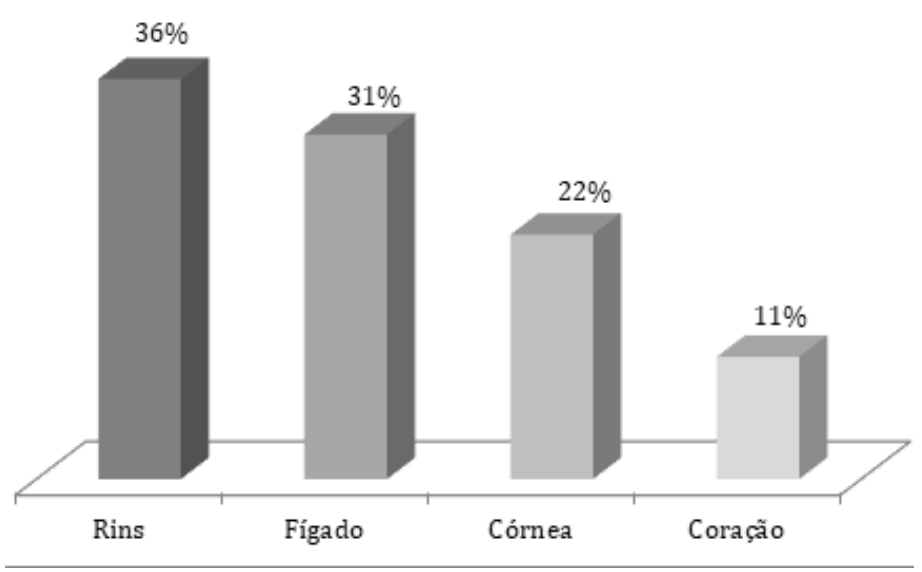

\section{RESULTADOS}

De 41 diagnósticos de morte encefálica, 34 potenciais doadores corresponderam aos critérios de inclusão da pesquisa, dos quais 15 (44\%) efetivaram a doação de órgãos. De acordo com o perfil constatado, predominou o traumatismo cranioencefálico, o sexo masculino e a faixa etária de 41 à 50 anos (Tabela 1). Os órgãos mais captados para doação foram rins e fígado, e não houve transplante pulmonar (Figura 1).

As alterações clínicas dos doadores de órgãos foram sintetizadas na Tabela 2: a elevação da creatinina e do GGT, assim como a hipernatremia e os distúrbios do potássio estão presentes na maior parte do grupo. $80 \%$ daqueles com creatinina elevada ( $N=8 / 10)$ tornaram-se doadores de rins; e $64 \%$ daqueles com hipernatremia $(N=7 / 11)$, de fígado. Todos os doadores de órgãos estavam em uso de drogas vasoativas, sendo noradrenalina a principal droga utilizada. Dividiu-se $100 \%$ noradrenalina, $27 \%$ vasopressina, $7 \%$ dopamina e $0 \%$ em epinefrina e dobutamina.

Os dados expõem que o balanço hídrico foi altamente positivo, tanto no primeiro quanto no segundo dia de avaliação do potencial doador falecido (Tabela 3). Durante o período total de permanência na UTI, o balanço maior que $1000 \mathrm{~mL}$, por dia, foi observado em $55 \%$ dos casos. O intervalo de tempo entre o diagnóstico de morte encefálica e a efetivação da doação resultou em um período máximo de dois dias de observação. Quatro $(11,76 \%)$ desenvolveram diabetes insipidus (diurese superior a $4 \mathrm{~mL} / \mathrm{kg} /$ hora e natremia superior a $160 \mathrm{~mL} / \mathrm{dl}$ ). 
Tabela 1: Dados descritivos dos potenciais doadores internados na Unidade de Terapia Intensiva

\begin{tabular}{lc}
\hline \multicolumn{1}{c}{ Variáveis } & Potencial Doador N (\%) \\
\hline Total & $34(100)$ \\
Masculino & $25(74)$ \\
Idade (anos) & \\
$<20$ & $5(15)$ \\
$21-30$ & $6(18)$ \\
$31-40$ & $8(24)$ \\
$41-50$ & $10(29)$ \\
$51-60$ & $2(6)$ \\
$>60$ & $3(9)$ \\
Motivo da internação & \\
TCE & $16(47)$ \\
AVC & $8(24)$ \\
HSA & $8(24)$ \\
Meningite & $1(3)$ \\
Hidrocefalia & $1(3)$ \\
Doadores & $15(44)$ \\
\hline
\end{tabular}

Tabela 2: Perfil clínico dos doadores de órgãos

\begin{tabular}{lc}
\hline \multicolumn{1}{c}{ Variáveis } & Doador de Órgãos N (\%) \\
\hline Total & $15(100)$ \\
Perfil renal & \\
Ureia $(>58 \mathrm{mg} / \mathrm{dL})$ & $6(40)$ \\
Creatinina $(>1,25 \mathrm{mg} / \mathrm{dL})$ & $10(67)$ \\
Perfil hepático & \\
TGO (>59 mg/dL) & $7(47)$ \\
TGP $(>72 \mathrm{mg} / \mathrm{dL})$ & $5(33)$ \\
BT $(>1,3 \mathrm{mg} / \mathrm{dL})$ & $1(7)$ \\
BI $(>1,1 \mathrm{mg} / \mathrm{dL})$ & $0(0)$ \\
FA $(>126 \mathrm{mg} / \mathrm{dL})$ & $2(13)$ \\
GGT $(>73 \mathrm{mg} / \mathrm{dL})$ & $10(67)$ \\
INR $(>1,4)$ & $2(13)$ \\
Perfil eletrolítico & \\
Sódio $(>145 \mathrm{mg} / \mathrm{dl})$ & $11(73)$ \\
Potássio $(<3,6$ ou $>5,0 \mathrm{mg} / \mathrm{dl})$ & $10(67)$ \\
Droga Vasoativa & Em uso \\
Noradrenalina & $15(100 \%)$ \\
Dobutamina & $0(0 \%)$ \\
Dopamina & $1(7 \%)$ \\
Vasopressina & $4(27 \%)$ \\
\hline
\end{tabular}

Tabela 3: Balanço hídrico após o diagnóstico de morte encefálica

\begin{tabular}{cc}
\hline \multicolumn{1}{c}{ Balanço Hídrico } & Potencial Doador N (\%) \\
\hline Dia 1 & 34 \\
$<0 \mathrm{ml} / \mathrm{dia}$ & $10(29)$ \\
$0-1000 \mathrm{ml} / \mathrm{dia}$ & $8(24)$ \\
$>1000 \mathrm{ml} / \mathrm{dia}$ & $16(47)$ \\
Dia 2 & 26 \\
$<0 \mathrm{ml} / \mathrm{dia}$ & $3(12)$ \\
$0-1000 \mathrm{ml} / \mathrm{dia}$ & $6(23)$ \\
$>1000 \mathrm{ml} / \mathrm{dia}$ & $17(65)$ \\
\hline
\end{tabular}

\section{DISCUSSÃO}

O controle hemodinâmico é fator chave nesse contexto e, portanto, a ressuscitação volêmica e as drogas vasoativas protagonizam na manutenção do doador falecido. A instituição do manejo sistematizado é consolidada nesse quesito e é capaz de aumentar a oferta de doadores em $82 \% .^{6}$ Atualmente, no Brasil, cerca de $10 \%$ dos potenciais doadores são perdidos devido ao colapso cardiocirculatório. ${ }^{1}$

Entretanto, a restrição da administração de fluidos e o uso de diuréticos podem beneficiar enxertos pulmonares. $^{5,9}$ De fato, a evidência mostra que o balanço hídrico mais próximo de zero, ajustado para otimização pulmonar, não afeta o resultado centrado ao receptor renal (disfunção precoce do enxerto). ${ }^{12}$

Miñambres et al, em seu artigo de 2010, discutem: "É geralmente assumido que pacientes submetidos ao transplante renal devem estar sob sobrecarga de fluidos. Assim, sugere-se que pressões centrais relativamente altas (pressão venosa central entre 10 e $15 \mathrm{mmHg}$ ) relacionam-se com uma melhor função precoce do enxerto, mas nenhum grande estudo prospectivo foi realizado". ${ }^{9}$ A tentativa de garantir pressão de perfusão orgânica e oxigenação adequadas através da euvolemia, ou até mesmo a hipervolemia, é transferida também ao doador. Apesar disso, o regime restritivo de volume (pressão venosa central menor que $6 \mathrm{mmHg}$ ) não apresentou prejuízo ao receptor renal. $^{9}$

De fato, a adequação ativa da euvolemia (positivamente ou negativamente) é crucial: tanto o uso de diuréticos quanto da desmopressina (análogo da vasopressina) foram associados com melhor desfecho à oferta de órgãos. ${ }^{5}$ 
A lesão das estruturas encefálicas hipotalâmicas pode conduzir à deficiência do hormônio antidiurético, a qual atua a favor da hipovolemia, hiperosmolaridade e hipernatremia. Teoricamente, a ressuscitação hormonal contribui para a restauração do volume circulante efetivo reduzindo a necessidade de catecolaminas. O uso concomitante da ressuscitação hormonal e da restrição do balanço hídrico (pressão venosa central menor que $10 \mathrm{mmHg}$ ) otimizou a oferta de pulmões e coração sem comprometer o transplante dos demais órgãos. ${ }^{11}$

Apesar desses achados, é preferível optar por uma estratégia sistêmica - em detrimento do sistema respiratório - em centros que não atuam no transplante de pulmão, aumentando a oferta de volume na manutenção hemodinâmica. ${ }^{13}$

Os objetivos clínicos são importantes ferramentas no manejo desse grupo: no cuidado com doadores marginais, a progressão dos parâmetros para a faixa preconizada foi associada a um melhor rendimento de órgãos doados por vítima. ${ }^{14}$ Apesar de não haver estudos prospectivos randomizados, as diretrizes recomendam a manutenção da pressão arterial média acima de 60$70 \mathrm{mmHg}$, débito urinário $1-3 \mathrm{~mL} / \mathrm{kg} / \mathrm{h}$ ora, otimização contínua das drogas vasoativas, monitorização da pressão venosa central (responsividade a volume) e fração de ejeção maior que $45 \%{ }^{4,13}$ Essa estratégia garante a pressão de perfusão orgânica e oxigenação adequadas com o mínimo de drogas vasoativas.

O monitoramento dinâmico do sistema circulatório, variação da pressão de pulso e índice cardíaco, direcionado como guia em um protocolo sistematizado de manejo hemodinâmico não provou superioridade no desfecho do transplante de órgãos (aumento da oferta e diminuição da disfunção dos órgãos no receptor). ${ }^{10}$

Trata-se do maior ensaio clínico randomizado sobre adequação do balanço hídrico com 556 doadores designados. Apesar da correlação positiva do monitoramento dinâmico em ensaios experimentais na minimização da resposta inflamatória da morte encefálica, o controle rigoroso do sistema cardiocirculatório realizado através da avaliação convencional é igualmente eficaz na otimização do doador de órgãos. ${ }^{10,15}$

Em uma revisão de ensaios randomizados no tema, de 87 trabalhos, 13 continham estratégias para o manejo hemodinâmico do potencial doador. ${ }^{16}$ Embora tenham sido constatados resultados insignificantes (hormônio tireoidiano e coloides), a dopamina e os análogos da vasopressina destacaram-se em termos de factualidade. ${ }^{16} \mathrm{O}$ tratamento com dose baixa de dopamina $(<4-5 \mu \mathrm{g} / \mathrm{kg} / \mathrm{min})$ melhorou o desfecho no pós-transplante do receptor renal (diminuição rápida da creatinina sérica e diálise durante a primeira semana)..$^{17,18}$

Os vasoativos são recomendados quando a ressuscitação volêmica falha em alcançar os objetivos hemodinâmicos. ${ }^{13} \mathrm{Em}$ comparação a outras UTI, o uso de drogas vasoativas esteve presente em 5889.2\%. ${ }^{5,9,19} \mathrm{Em}$ um estudo retrospectivo recente, as drogas dividiram-se em: $60 \%$ dopamina, $49 \%$ noradrenalina, $11 \%$ dobutamina e $3 \%$ adrenalina dos potenciais doadores. ${ }^{19}$ Novos estudos prospectivos são necessários para aperfeiçoar o uso da noradrenalina no controle hemodinâmico na morte encefálica e no desfecho do transplante de órgãos.

Em uma análise multicêntrica com 14125 doadores falecidos, observou-se, em média, creatinina de 1,32 $\mathrm{mg} / \mathrm{dL}$ e a natremia de $146,41 \mathrm{mg} / \mathrm{dL}$, semelhante aos resultados deste trabalho. ${ }^{5} \mathrm{Em}$ virtude de um possível efeito danoso da hipernatremia ao funcionamento do enxerto hepático pós-transplante, é recomendável corrigir ativamente esse distúrbio. . $^{13,20}$

A efetivação da doação ocorreu em $34.3 \%$ de 469 potenciais doadores, em um centro de trauma na América do Norte. ${ }^{6}$ Os dados de 2018 da Associação Brasileira de Transplante de Órgãos correspondem a uma efetivação média por notificação, em cerca de 33\% no Brasil. ${ }^{1}$

\section{CONCLUSÃO}

Apesar da hiper-hidratação, a efetivação dos transplantes tem resultado acima da média neste centro. A permissividade do balanço hídrico fortemente positivo é justificada pela ausência de centro especializado no transplante pulmonar na região. $O$ controle rigoroso do perfil hemodinâmico do potencial doador de órgãos aumenta a viabilidade do transplante e deve ser adaptado e sistematizado à realidade de cada localidade. 


\section{ABSTRACT}

Introduction: The aggressive and effective approach to the potential donor benefits multiple organ transplantation both quantitatively and qualitatively. In view of the physiological changes in brain death, hemodynamic balance plays an important role in optimizing this group. Purpose: to assess the fluid balance and other parameters of hemodynamic profile of patients diagnosed with brain death in ICU related to clinical setting of multiple organ transplantation. Methods: descriptive, retrospective and cross-sectional study of patients diagnosed with brain death from 2013 to 2014 in an ICU trauma reference center in Pernambuco. Data were systematically extracted from patients' medical records, and the variables of general, clinical and fluid balance profile were synthesized by absolute and relative frequency. Results: Of 41 brain death diagnosis, 34 potential donors were analyzed, of which 15 (44\%) performed organ donation. Head trauma, male gender and ages from 41 to 50 years old were predominant in the group. Kidneys and liver were the most donated organs, and there was no lung transplantation. All were using vasoactive drugs and the fluid balance was highly positive on the first and second days of the potential deceased donor's assessment. Conclusion: Despite hyper hydration, effective organ donation has been above average in this center. The permissiveness of high positive fluid balance is justified by the absence of lung transplantation center in the region. Strict control of the hemodynamic profile of the potential organ donor increases the feasibility of the transplant and must be adapted and systematized to the reality of each location.

Keywords: Organ Donation, Organ Procurement, Transplantation, Brain Death, Fluid Balance.

\section{REFERÊNCIAS}

1. RBT 2018 (JAN/DEZ) - ABTO, Dimensionamento dos Transplantes no Brasil e em cada Estado (2011-2018), páginas 22 e 25. p.:1-89.

2. Watts RP, Thom O, Fraser JF. Inflammatory signalling associated with brain dead organ donation: from brain injury to brain stem death and posttransplant ischaemia reperfusion injury. J Transplant. 2013:521369.

3. Moreira WC, Barbosa TMA, Ribeiro WR de A, Damasceno CS, Alencar D de C, Vieira SF. Assistência de enfermagem no processo de doação de órgãos e transplantes. Rev Prev Infecção E Saúde. 2016;2(1-2):32-42.

4. Westphal GA, Caldeira Filho M, Vieira KD, Zaclikevis VR, Bartz MCM, Wanzuita R, et al. Guidelines for potential multiple organ donors (adult): part I. Overview and hemodynamic support. Rev Bras Ter Intensiva. 2011;23(3):255-68.

5. Selck FW, Deb P, Grossman EB. Deceased Organ Donor Characteristics and Clinical Interventions Associated with Organ Yield. Am J Transplant. 2008;8(5):965-74.

6. Salim A, Velmahos GC, Brown C, Belzberg H, Demetriades D. Aggressive Organ Donor Management Significantly Increases the Number of Organs Available for Transplantation. J Trauma Acute Care Surg. 2005;58(5):991-4.
7. Maciel CB, Greer DM. ICU Management of the Potential Organ Donor: State of the Art. Curr Neurol Neurosci Rep. 2016;16(9):86.

8. Barklin A. Systemic inflammation in the brain-dead organ donor. Acta Anaesthesiol Scand. 2009;53(4):425-35.

9. Miñambres E, Rodrigo E, Ballesteros MA, Llorca J, Ruiz JC, Fernandez-Fresnedo G, et al. Impact of restrictive fluid balance focused to increase lung procurement on renal function after kidney transplantation. Nephrol Dial Transplant. 2010;25(7):2352-6.

10. Al-Khafaji A, Elder M, Lebovitz DJ, Murugan R, Souter $M$, Stuart S, et al. Protocolized fluid therapy in brain-dead donors: the multicenter randomized MOnIToR trial. Intensive Care Med. 2015;41(3):418-26.

11. Abdelnour T, Rieke S. Relationship of hormonal resuscitation therapy and central venous pressure on increasing organs for transplant. J Heart Lung Transplant Off Publ Int Soc Heart Transplant. 2009;28(5):480-5.

12. Miñambres E, Ballesteros MA, Rodrigo E, García-Miguélez A, Llorca J, Ruiz JC, et al. Aggressive lung donor management increases graft procurement without increasing renal graft loss after transplantation. Clin Transplant. 2013;27(1):52-9. 
Balanço hídrico no potencial doador de múltiplos órgãos em centro de referência de trauma.

13. Kotloff RM, Blosser S, Fulda GJ, Malinoski D, Ahya VN, Angel $L$, et al. Management of the Potential Organ Donor in the ICU: Society of Critical Care Medicine/American College of Chest Physicians/Association of Organ Procurement Organizations Consensus Statement. Crit Care Med. 2015;43(6):1291-325.

14. Patel MS, Zatarain J, Cruz SDL, Sally MB, Ewing T, Crutchfield M, et al. The Impact of Meeting Donor Management Goals on the Number of Organs Transplanted per Expanded Criteria Donor: A Prospective Study From the UNOS Region 5 Donor Management Goals Workgroup. JAMA Surg. 2014;149(9):969-75.

15. Murugan R, Venkataraman R, Wahed AS, Elder M, Carter M, Madden NJ, et al. Preload responsiveness is associated with increased interleukin- 6 and lower organ yield from brain-dead donors. Crit Care Med. 2009;37(8):2387-93.

16. Dikdan GS, Mora-Esteves C, Koneru B. Review of randomized clinical trials of donor management and organ preservation in deceased donors: opportunities and issues. Transplantation. 2012;94(5):425-41.
17. Schnuelle P, Yard BA, Braun C, Dominguez-Fernandez E, Schaub M, Birck R, et al. Impact of Donor Dopamine on Immediate Graft Function after Kidney Transplantation. Am J Transplant. 2004;4(3):419-26.

18. Schnuelle P, Gottmann U, Hoeger S, Boesebeck D, Lauchart W, Weiss C, et al. Effects of donor pretreatment with dopamine on graft function after kidney transplantation: a randomized controlled trial. JAMA. 2009;302(10):1067-75.

19. Birtan D, Arslantas MK, Altun GT, Dincer PC, Gecegormez S, Demirel A, et al. Effect of Vasoactive Therapy Used for Brain-Dead Donors on Graft Survival After Kidney Transplantation. Transplant Proc. 2018;50(5):1289-91.

20. Totsuka E, Dodson F, Urakami A, Moras N, Ishii T, Lee $M-C$, et al. Influence of high donor serum sodium levels on early postoperative graft function in human liver transplantation: Effect of correction of donor hypernatremia. Liver Transpl Surg. 1999;5(5):421-8. 